\title{
Electron Trapping at Point Defects on Hydroxylated Silica Surfaces
}

\author{
Livia Giordano, ${ }^{1}$ Peter V. Sushko, ${ }^{2}$ Gianfranco Pacchioni, ${ }^{1}$ and Alexander L. Shluger ${ }^{2}$ \\ ${ }^{1}$ Dipartimento di Scienza dei Materiali, Università di Milano-Bicocca, via R. Cozzi, 53-20125 Milano, Italy \\ ${ }^{2}$ Department of Physics and Astronomy, London Centre for Nanotechnology, Materials Simulation Laboratory, \\ University College London, Gower Street, London WC1E 6BT, United Kingdom
}

(Received 5 April 2007; published 24 September 2007)

\begin{abstract}
The origin of electron trapping and negative charging of hydroxylated silica surfaces is predicted based on accurate quantum-mechanical calculations. The calculated electron affinities of the two dominant neutral paramagnetic defects, the nonbridging oxygen center, $\equiv \mathrm{Si}-\mathrm{O}^{\bullet}$, and the silicon dangling bond, $\equiv \mathrm{Si}^{\bullet}$, demonstrate that both defects are deep electron traps and can form the corresponding negatively charged defects. We predict the structure and optical absorption energies of these diamagnetic defects.
\end{abstract}

DOI: 10.1103/PhysRevLett.99.136801

The mechanisms of electron and hole trapping in oxides, as well as the nature of trapping sites, are important in a wide range of phenomena and applications. In some of them, such as scanning electron microscopy and electron lithography, charging is considered as a source of artifacts. It may have dramatic consequences for the performance and reliability of, for example, microelectronic devices containing interfaces of metal oxides and semiconductors, optical fibers, and in corrosion protection. In other applications, such as the formation of long-lifetime electrets [1] or ultrashort pulsed laser ablation [2], charging is the base of the process itself. Electron and hole formation and subsequent trapping may be responsible for the dramatic electrostatic and optical phenomena that are observed during seismic activity [3]. Charge trapping is important for understanding Martian saltation, tribo-electric charging, and discharge phenomena, while dust particle charging and adhesion can become a nuisance in future Mars missions [4]. Surface charging and $\mathrm{x}$-ray emission from insulator surfaces induced by collisions with highly charged ions is of relevance to cometary and planetary spectroscopy [5]. Taking into account its abundance and technological importance, it is not surprising that $\mathrm{SiO}_{2}$ plays a key role in all the above phenomena.

Whatever the area of interest, it is crucial to establish the specific trapping sites, their stable atomic configurations, and spectroscopic properties. A considerable amount of data correlating the positive charging of silica samples and their defect properties were obtained using spectroscopic methods [6-8]. However, identifying sites responsible for electron trapping in silica bulk and surface proved particularly challenging. Recently, microscopic contact charging and charge dissipation on and inside thin silicon oxide films has been demonstrated using a controllable contact charging method [9]. Dispersive charge transport along the silica surface has been observed by electrostatic force microscopy [10]. Single-electron tunneling force spectroscopy of an individual electronic state in a silica surface has been performed [11]. However, the nature of surface and bulk sites capable of trapping extra electrons has not yet been established.
PACS numbers: 73.20. $-\mathrm{r}, 71.15 . \mathrm{Mb}, 71.55 .-\mathrm{i}, 78.20 . \mathrm{Bh}$

The investigations of the charge trapping and release by defects are complicated because of a large number of possible charge redistribution channels; e.g., electrons and holes can be transferred, respectively, through the conduction and valence band states or indirectly through the diffusion of impurities such as protons, $\mathrm{Li}$ and $\mathrm{Na}$, etc. In addition, charge trapping can result in a dramatic structural reorganization of the defect site. For example, a positively charged oxygen vacancy, known as $E^{\prime}{ }_{1}$ in $\alpha$-quartz, and a neutral oxygen vacancy have very different structural arrangements [6,7]. Investigation of the electron and hole trapping at surfaces is even more problematic because of the lower concentration of surface defects and the overlap of their spectroscopic characteristics with those of bulk defects. Furthermore, relaxation channels at surfaces can be different from those in the bulk.

In this Letter, we demonstrate theoretically the possibility of electron trapping by two dominant point defects at silica surfaces $[12,13]$ : (i) the nonbridging oxygen center (NBO or $\equiv \mathrm{Si}-\mathrm{O}^{\bullet}$ ) and (ii) a three-coordinated silicon with a singly occupied dangling bond $\left(\equiv \mathrm{Si}^{\bullet}\right)$, a surface analog of the bulk $E^{\prime}$ center. These defects have been characterized by electron paramagnetic resonance (EPR) spectroscopy and optical absorption measurements [7] and can be created, for example, by mechanical or thermochemical activation. The finding that these defects can act as deep electron traps helps our understanding of the mechanisms of radiation-induced processes in silica samples, tribocharging, and seismo-electromagnetic phenomena. The presence of negatively charged defects on the silica surface can be of great importance also in determining the chemical properties of the material and that of deposited metal particles, including their optical response [13]. While the neutral analogs of surface NBO and $E^{\prime}$ centers can be detected using EPR spectroscopy, their charged states are EPR-silent and, thus, much more difficult to identify experimentally. We characterize each defect by calculating its electron affinity (EA), ionization potential (IP), and optical absorption energies, thus providing a basis for their experimental detection with optical spectroscopies. 
The defect properties have been calculated using an embedded cluster method, as implemented in the GUESS code [14], designed to study charge trapping by point defects [15] and the associated long-range relaxation and polarization effects. A silica surface is represented by a hemispherical nanocluster, where the oxygen-terminated surface has been saturated with $\mathrm{H}$ atoms to mimic hydroxylation. The nanocluster is then divided into a polarizable region I and a surrounding nonpolarizable region II. Region I includes (i) a defect site and surrounding treated quantummechanically (QM cluster), (ii) an interface region, formed by pseudo-Si atoms $\left(\mathrm{Si}^{*}\right)$ [15], connecting the QM cluster to the rest of the solid, and (iii) the remaining part, modeled using the shell model [16]. Region II contains only classical unpolarizable ions [15]. The total energy of the system is minimized with respect to all degrees of freedom in region I. The QM contribution to the total energy is calculated using the density functional theory (DFT), a hybrid B3LYP functional $[17,18]$, and Gaussian-type basis functions $\left(6-31 \mathrm{G}^{*}\right.$ on $\mathrm{Si}$ and $\mathrm{O}$ involved in the defect, $6-31 \mathrm{G}$ on the other $\mathrm{Si}$ and $\mathrm{O}$ atoms). Classical interatomic potentials [19] have been used to describe the interaction between lattice $\mathrm{Si}$ and $\mathrm{O}$ atoms. The $\mathrm{OH}$ groups in the classical part are described by a potential [20] modified to make it compatible with the Si-O potential of Ref. [19].

The vertical and adiabatic electron affinities $\left(\mathrm{EA}_{v}\right.$ and $\mathrm{EA}_{a}$, respectively) and ionization potentials $\left(\mathrm{IP}_{v}\right.$ and $\left.\mathrm{IP}_{a}\right)$ of the defect centers with respect to the vacuum level were calculated as the differences in total energies of the neutral and charged states, $\triangle \mathrm{SCF}$. In the calculation of vertical IP and EA, the nuclei are fixed, but the long-range electronic polarization is included, while the calculation of the adiabatic IP and EA involves the full electronic and ionic relaxation. The optical properties have been calculated using the time-dependent DFT approach (TD-DFT) [21].

We have considered hydroxylated surfaces of three silica polymorphs: edingtonite(010), $\beta$-cristobalite(111), and $\alpha$-quartz(0001). The concentration of silanol groups is lower on the edingtonite than on cristobalite and quartz surfaces. Variations in the structure of isolated silanols $(\equiv \mathrm{SiOH}$, as in edingtonite and cristobalite) and geminal silanols $\left[=\mathrm{Si}(\mathrm{OH})_{2}\right.$ as in quartz] provide a diversity of configurations that can be present on the surface of amorphous silica [12]. When not otherwise specified, the data refer to the edingtonite surface.

To estimate the position of the defect level in the band gap, one should know the positions of the valence band (VB) and conduction band (CB) edges with respect to the vacuum. These are not easy to measure experimentally nor to obtain theoretically since they depend on the surface morphology as well as on the degree of surface hydroxylation. A value of $10 \mathrm{eV}$ for the top of the oxide VB edge can be extrapolated from the data on the $\mathrm{SiO}_{2} / \mathrm{Si}$ interface [22]. This is close to the value of $10.3 \mathrm{eV}$ inferred from experimental data on bulk silica [23]. In the case of hydroxylated silica studied here, the $\mathrm{OH}$ groups create a dipole layer which can affect the position of the levels with respect to vacuum and add states at the top of the VB and at the bottom of the CB. Using a large QM cluster $\mathrm{H}_{5} \mathrm{Si}_{31} \mathrm{O}_{77} \mathrm{Si}_{25}^{*}$ (6-31G* basis set on all $\mathrm{O}$ and $\mathrm{Si}$ atoms), we find that the top of the VB of the hydroxylated edingtonite surface is $10.1 \mathrm{eV}$ below the vacuum level, whereas the bottom of the $\mathrm{CB}$ is $0.6 \mathrm{eV}$ below the vacuum level (see Fig. 1). These energies have been determined by removing and adding, respectively, an electron to the cluster and calculating the corresponding total energies: $E_{\triangle \mathrm{SCF}}(\mathrm{VB})=$ $E\left(\mathrm{SiO}_{2}^{+}\right)-E\left(\mathrm{SiO}_{2}\right) ; \quad E_{\Delta \mathrm{SCF}}(\mathrm{CB})=E\left(\mathrm{SiO}_{2}\right)-E\left(\mathrm{SiO}_{2}^{-}\right)$. Notice that the resulting hole and added electron are fully delocalized over the entire cluster, consistent with the VB and $\mathrm{CB}$ character of the corresponding electronic states. We can now define the position of the energy levels of the defects of interest by calculating their ionization potentials with respect to the vacuum level.

First, we consider the charge trapping by the NBO center, formed on the hydroxylated surface by removal of an $\mathrm{H}$ atom. The neutral center has a singly occupied nonbonding $\mathrm{O}(2 p)$ orbital of the nonbridging oxygen $\left(\mathrm{O}_{\mathrm{nb}}\right)$, Fig. 2(a). Its energy level is just above the top of the VB and can be readily occupied by an extra-electron $\left(\equiv \mathrm{Si}-\mathrm{O}^{\bullet}+e^{-} \rightarrow \equiv \mathrm{Si}^{-} \mathrm{O}^{-}\right): \mathrm{EA}_{v}$ and $\mathrm{EA}_{a}$ are $3.9 \mathrm{eV}$ and $5.3 \mathrm{eV}$, respectively, Table $\mathrm{I}$ and Fig. 1. In $\beta$-cristobalite and $\alpha$-quartz, $\mathrm{EA}_{a}$ is larger, $5.8 \mathrm{eV}$ (Table I) because of the electrostatic interaction of the charged $\equiv \mathrm{Si}^{-} \mathrm{O}^{-}$defect with the vicinal silanol groups which provide a kind of "solvation effect." Ionization (hole trapping) of a neutral NBO center is unlikely because

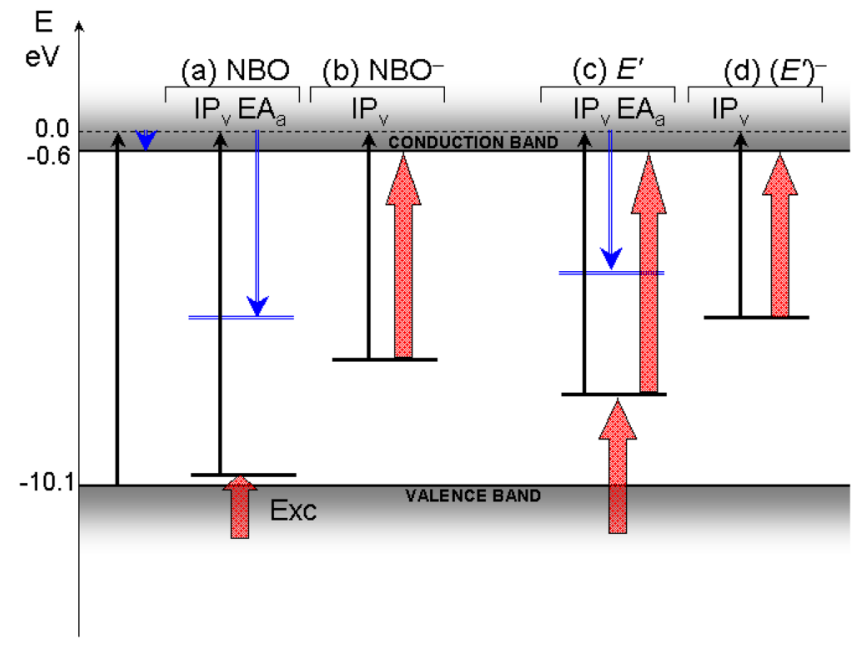

FIG. 1 (color online). Schematic representation of the nature of electron and hole trapping by $\mathrm{NBO}$ center $\left(\equiv \mathrm{Si}-\mathrm{O}^{\bullet}\right)$ (a) and (b) and $E^{\prime}$ center $\left(\equiv \mathrm{Si}^{\bullet}\right)$ (c) and (d), on the silica surface with formation of the corresponding $\equiv \mathrm{Si}-\mathrm{O}^{-}$and $\equiv \mathrm{Si}^{-}$charged defects. Large arrows indicate the nature of the electronic excitations (Exc) of the defects. In the neutral variants, $\equiv$ $\mathrm{Si}-\mathrm{O}^{\bullet}$ and $\equiv \mathrm{Si}^{\bullet}$, excitations take place from the $\mathrm{VB}$ to the singly occupied defect states in the gap or from this level and the $\mathrm{CB}$; in the negatively charged variants, $\equiv \mathrm{Si}_{-} \mathrm{O}^{-}$and $\equiv \mathrm{Si}^{-}$, the corresponding states are doubly occupied, and transitions can only occur from these levels to the CB. 


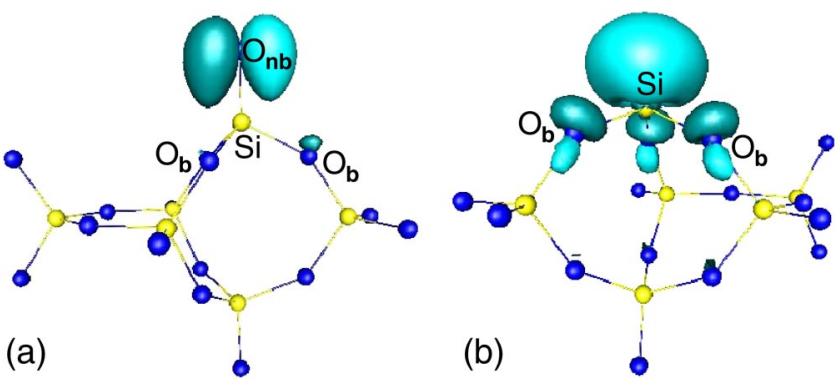

FIG. 2 (color online). Highest occupied molecular orbitals in (a) $\mathrm{NBO}$ center $\left(\equiv \mathrm{Si}-\mathrm{O}^{\bullet}\right)$ and $\left(\right.$ b) $E^{\prime}$ center $\left(\equiv \mathrm{Si}^{\bullet}\right)$ on the surface of silica. These orbitals are singly occupied in the neutral (paramagnetic) state and doubly occupied in the negatively charged state of the defect.

the filled states in the gap are just at the top of the VB $\left(\mathrm{IP}_{v}\right.$ of $\mathrm{NBO} \approx 11.0 \mathrm{eV}$, Fig. 1). In this respect, the behavior of $\equiv \mathrm{Si}-\mathrm{O}^{\bullet}$ defects at silica surfaces does not differ from that of the corresponding bulk centers or of the regular sites.

The capture of one electron by the NBO center is accompanied by a relaxation energy of $1.5-2 \mathrm{eV}$ and a strong local lattice distortion. The $\mathrm{Si}-\mathrm{O}_{\mathrm{nb}}$ distance shrinks from 1.66 to $1.54 \AA$, and the $\mathrm{O}_{b}-\mathrm{Si}_{-} \mathrm{O}_{b}$ angles decrease on average from $108^{\circ}$ to $100^{\circ}$. This shortening of the $\mathrm{Si}-\mathrm{O}_{\mathrm{nb}}$ distance, experimentally inferred in alkali silicate glasses, indicates that the $\mathrm{O}_{\mathrm{nb}}(2 p)$ state, which accommodates the extra-electron, has a bonding interaction with the $\mathrm{Si}$ atom.

The electronic states of the $\equiv \mathrm{Si}-\mathrm{O}^{-}$defect $\left(\mathrm{NBO}^{-}\right)$ include two almost degenerate nonbonding $\mathrm{O}_{\mathrm{nb}}(2 p)$ orbitals at about $2.3 \mathrm{eV}$ and a bonding $\sigma$ orbital at about $0.6 \mathrm{eV}$ above the top of the VB, respectively. The energies of these orbitals are shifted up with respect to the neutral case due to the increased Coulomb repulsion, Fig. 1. The electron trapping at the NBO center alters the nature of its optical absorption. In particular, the transitions from the VB to the unoccupied $2 p$ state, characteristic of the neutral NBO $[12,24]$, disappear. Instead, the $\mathrm{NBO}^{-}$center is characterized by an intense absorption band at $6.0 \mathrm{eV}$ due to a transition from the doubly occupied $\mathrm{O}_{\mathrm{nb}}(2 p)$ orbitals to the $\mathrm{CB}$, Fig. 1. We note that this absorption band of $\mathrm{NBO}^{-}$ is in the energy range of the recently observed band at 6.4$6.8 \mathrm{eV}$ attributed to bulk NBO $[25,26]$. The calculated ionization energy of $\mathrm{NBO}^{-}$is $6.5 \mathrm{eV}$ in edingtonite (see Table I). Given only a $0.5 \mathrm{eV}$ difference between the excitation and the ionization energies, we expect that photo-excitation of $\mathrm{NBO}^{-}$results in its thermal ionization. The IP of $\mathrm{NBO}^{-}$in cristoabalite and quartz, 7.3 and $7.8 \mathrm{eV}$, respectively, are noticeably larger due to the solvation by the neighboring $\mathrm{OH}$ groups.

Various mechanisms can lead to the formation of $\mathrm{NBO}^{-}$ surface defects, such as charging the neutral precursor with an electron gun, doping with alkali atoms or other electron donors, or by radiation-induced proton transfer:

$$
\equiv \mathrm{Si}-\mathrm{OH}+\equiv \mathrm{Si}-\mathrm{O}-\mathrm{Si} \equiv \rightarrow \equiv \mathrm{Si}_{-} \mathrm{O}^{-}+\equiv \mathrm{Si}_{-} \mathrm{OH}^{+}-\mathrm{Si} \equiv .
$$

The energy change associated to reaction (1) was estimated for edingtonite, where the proton is transferred from an isolated hydroxyl group, $\equiv \mathrm{Si}-\mathrm{OH}$, to a vicinal bridging oxygen, $\equiv \mathrm{Si}-\mathrm{O}-\mathrm{Si} \equiv$. The cost of the proton transfer is about $2 \mathrm{eV}$, an energy value readily accessible under radiation or mechanical stress conditions, and the resulting pairs of defects are metastable.

We turn now to the $\equiv \mathrm{Si}^{\bullet}$ surface center. This can be formally obtained on a hydroxylated surface by breaking a $\equiv \mathrm{Si}-\mathrm{OH}$ bond. In the neutral state of this paramagnetic defect, a single-electron occupies a $s p^{3}$ orbital of the threecoordinated silicon, Fig. 2(b), at $1.6 \mathrm{eV}$ above the VB. EA $v$ of this center is $\approx 2.5 \mathrm{eV}$, and increases to $4 \mathrm{eV}$ after the lattice relaxation is taken into account, Table I and Fig. 1. In $\equiv \mathrm{Si}^{-}$, the $\mathrm{Si}_{3 \mathrm{C}}$ atom moves outwards, resulting in elongation of $\mathrm{Si}-\mathrm{O}$ distances by $0.11 \AA$, while the average $\mathrm{O}_{b}-\mathrm{Si}-\mathrm{O}_{b}$ angle decreases from $108^{\circ}$ to $95^{\circ}$. After electron trapping and consequent relaxation, the $\mathrm{Si}\left(s p^{3}\right)$ lone pair state shifts to higher energy, Fig. 1, and is found $2.5 \mathrm{eV}$ above the top of the VB. The energies of the $2 p$ orbitals of the three oxygen atoms next to $\mathrm{Si}_{3 \mathrm{C}}$ are pushed toward the top of the VB by the Coulomb repulsion. Similarly to NBO, the vertical EAs have close values for the three surfaces, while, upon relaxation, the interaction with the vicinal silanol in $\alpha$-quartz makes an additional contribution to the EA. The negatively charged $\equiv \mathrm{Si}^{-}$center in edingtonite has an optical absorption band at $5.2 \mathrm{eV}$ due to a transition from the doubly occupied state to the $\mathrm{CB}$,

TABLE I. Vertical and adiabatic electron affinities $\left(\mathrm{EA}_{v}\right.$ and $\left.\mathrm{EA}_{a}\right)$ of the $\equiv \mathrm{Si}-\mathrm{O}^{\bullet}$ and $\equiv \mathrm{Si}^{\bullet}$ defect centers and vertical ionization potential $\left(\mathrm{IP}_{v}\right)$ of the $\equiv \mathrm{Si}-\mathrm{O}^{-}$and $\equiv \mathrm{Si}^{-}$defect centers formed on hydroxylated silica surfaces.

\begin{tabular}{lccc}
\hline \hline \multicolumn{1}{c}{$\equiv \mathrm{Si}-\mathrm{O}^{\bullet}$} & $\mathrm{EA}_{v}\left(\equiv \mathrm{Si}-\mathrm{O}^{\bullet}\right), \mathrm{eV}$ & $\mathrm{EA}_{a}\left(\equiv \mathrm{Si}-\mathrm{O}^{\bullet}\right), \mathrm{eV}$ & $\mathrm{IP}_{v}\left(\equiv \mathrm{Si}-\mathrm{O}^{-}\right), \mathrm{eV}$ \\
\hline edingtonite(010) & 3.89 & 5.31 & 6.50 \\
$\beta$-cristobalite(111) & 4.03 & 5.77 & 7.32 \\
$\alpha$-quartz(0001) & 3.78 & 5.78 & 7.79 \\
$\quad \equiv \mathrm{Si}^{\bullet}$ & $\mathrm{EA}_{v}(\equiv \mathrm{Si} \bullet), \mathrm{eV}$ & $\mathrm{EA}_{a}(\equiv \mathrm{Si} \bullet), \mathrm{eV}$ & $\mathrm{IP}_{v}\left(\equiv \mathrm{Si}^{-}\right), \mathrm{eV}^{-3}$ \\
edingtonite$(010)$ & 2.53 & 3.93 & 5.30 \\
$\beta$-cristobalite(111) & 2.76 & 4.37 & 6.02 \\
$\alpha$-quartz(0001) & 2.40 & 4.50 & 6.29 \\
\hline \hline
\end{tabular}

${ }^{a} \mathrm{QM}$ clusters: $\mathrm{HSi}_{6} \mathrm{O}_{15} \mathrm{Si}_{7}^{*}$ for edingtonite, $\mathrm{HSi}_{10} \mathrm{O}_{28} \mathrm{Si}_{15}^{*}$ for $\beta$-cristobalite(111), and $\mathrm{H}_{2} \mathrm{Si}_{6} \mathrm{O}_{18} \mathrm{Si}_{9}^{*}$ for $\alpha$-quartz(0001). 
Fig. 1. This excitation energy is similar to the $\mathrm{IP}_{v}, 5.3 \mathrm{eV}$, suggesting that photo-excitation of this defect can lead to its ionization, similarly to the case of $\mathrm{NBO}^{-}$. As for the $\equiv \mathrm{Si}-\mathrm{O}^{-}$center, also for $\equiv \mathrm{Si}^{-}$, we found that $\mathrm{IP}_{v}$ is about $1 \mathrm{eV}$ larger for cristobalite and quartz surfaces, Table I. The calculated $\mathrm{IP}_{v}$ of the $\equiv \mathrm{Si}^{\bullet}$ neutral defect, $\approx 9 \mathrm{eV}$, is very high and the resulting $\mathrm{Si}_{3 \mathrm{C}}^{+}$center has an $s p^{2}$ hybridization and, together with the three $\mathrm{O}$ atoms, forms a trigonal planar structure.

We consider now possible interactions on the silica surface involving the charge trapping centers described in this Letter. The interaction between $\equiv \mathrm{Si}^{-}$and a neighboring silanol group could result in the proton transfer:

$$
\equiv \mathrm{Si}^{-}+\equiv \mathrm{Si}-\mathrm{OH} \rightarrow \equiv \mathrm{Si}-\mathrm{H}+\equiv \mathrm{Si}_{-} \mathrm{O}^{-} .
$$

In the case of $\alpha$-quartz surface, this reaction is exothermic by about $1 \mathrm{eV}$, as the consequence of the lower EA of $\equiv \mathrm{Si}^{\bullet}$ with respect to $\equiv \mathrm{Si}-\mathrm{O}^{\bullet}$ and of the comparable strengths of the Si-O-H and $\mathrm{Si}-\mathrm{H}$ bonds (about $5 \mathrm{eV}$ ). Thus, thermodynamic considerations indicate that protonation of $\equiv \mathrm{Si}^{-}$centers can occur on hydroxylated surfaces with formation of $\equiv \mathrm{Si}-\mathrm{O}^{-}$defects.

We have seen above that $\mathrm{EA}_{a}$ of the NBO center in edingtonite is $5.3 \mathrm{eV}$ while $\mathrm{IP}_{a}$ of $E^{\prime}$ is $7.5 \mathrm{eV}$. Thus, the net cost for the charge disproportionation process between these defects

$$
\equiv \mathrm{Si}^{\bullet}+\equiv \mathrm{Si}-\mathrm{O}^{\bullet} \rightarrow \equiv \mathrm{Si}^{+}+\equiv \mathrm{Si}^{-} \mathrm{O}^{-}
$$

is of $2.2 \mathrm{eV}$. If the two resulting charged defects, $\equiv \mathrm{Si}^{+}$and $\equiv \mathrm{Si}-\mathrm{O}^{-}$, are sufficiently close, their electrostatic interaction could stabilize this state.

In conclusion, our calculations demonstrate that two well-known paramagnetic centers of the silica surface, the $\mathrm{NBO}\left(\equiv \mathrm{Si}-\mathrm{O}^{\bullet}\right)$ and $E^{\prime}\left(\equiv \mathrm{Si}^{\bullet}\right)$ are deep electron traps able to form stable negatively charged surface centers, which can contribute to negative charging of silica surfaces, with important consequences on their chemical and physical properties [9-11]. Furthermore, the formation of the diamagnetic $\mathrm{NBO}^{-}$defect by electron trapping explains the absence of correlation between the amount of hydrogen desorbed from high-OH silica under irradiation with $7.9 \mathrm{eV}$ photons and the number of paramagnetic NBO centers detected [27]. Our results also suggest that similar charge trapping can readily occur on qualitatively similar neutral NBO and $E^{\prime}$ bulk centers, and provide a simple mechanism to electrically compensate the formation of hole traps [28,29]. Finally, we note that for thin silica films grown on metal substrates (e.g. Mo) [30], surface defects can be charged due to electron tunneling from the substrate material [31]. Indeed, comparing energies given in Table I with typical metal work functions $(4.5 \mathrm{eV})$, shows that electrons can tunnel into $\mathrm{NBO}$ and possibly $\equiv \mathrm{Si}^{\bullet}$ type defects forming the negatively charged centers.
This work was supported by the Italian MIUR through a Cofin project. L. G. thanks EPSRC for support under Grant No. GR/S80080/1. P. V. S. is supported by the Grant-in-Aid for Creative Scientific Research (No. 16GS0205) from the Japanese Ministry of Education, Culture, Sports, Science and Technology. We are grateful to A. V. Kimmel for helpful discussions.

[1] G. M. Sessler and J.E. West, Appl. Phys. Lett. 17, 507 (1970).

[2] R. Stoian et al., Phys. Rev. Lett. 88, 097603 (2002).

[3] A. Takeuchi, H. Nagahama, and T. Hashimoto, Phys. Chem. Earth 29, 359 (2004).

[4] F. B. Gross, J. Electrost. 58, 147 (2003).

[5] N. Djurić et al., Astrophys. J. 635, 718 (2005).

[6] L. Skuja, in Defects in $\mathrm{SiO}_{2}$ and Related Dielectrics: Science and Technology, edited by G. Pacchioni, L. Skuja, and D.L. Griscom, NATO Science Series (Kluwer, Dordrecht, 2000), p. 73.

[7] V. A. Radzig in Ref. [6], p. 339.

[8] D. L. Griscom, J. Non-Cryst. Solids 352, 2601 (2006).

[9] S. Morita and Y. Sugawara, Thin Solid Films 393, 310 (2001).

[10] J. Lambert et al., Phys. Rev. B 71, 155418 (2005).

[11] E. Bussmann and C.C. Williams, Appl. Phys. Lett. 88, 263108 (2006).

[12] L. Giordano et al., Phys. Rev. B 75, 024109 (2007).

[13] J.-M. Antonietti et al., Phys. Rev. Lett. 94, 213402 (2005).

[14] P. V. Sushko, A. L. Shluger, and C. R. A. Catlow, Surf. Sci. 450, 153 (2000).

[15] V. B. Sulimov et al., Phys. Rev. B 66, 024108 (2002).

[16] B. G. Dick and A. W. Overhauser, Phys. Rev. 112, 90 (1958).

[17] A. D. Becke, J. Chem. Phys. 98, 5648 (1993).

[18] C. Lee, W. Yang, and R. G. Parr, Phys. Rev. B 37, 785 (1988).

[19] B. W. H. van Beest, G. J. Kramer, and R. A. van Santen, Phys. Rev. Lett. 64, 1955 (1990).

[20] J. Du and A. N. Cormack, J. Am. Ceram. Soc. 88, 2532 (2005).

[21] M. J. Frisch et al., computer code GAUSSIAN 03, Gaussian, Pittsburgh, PA, 2003.

[22] F. J. Himpsel et al., Phys. Rev. B 38, 6084 (1988).

[23] D. L. Griscom, J. Non-Cryst. Solids 24, 155 (1977).

[24] T. Suzuki et al., Phys. Rev. Lett. 90, 186404 (2003).

[25] H. Hosono et al., Solid State Commun. 122, 117 (2002).

[26] M. Cannas and F. M. Gelardi, Phys. Rev. B 69, 153201 (2004).

[27] H. Nishikawa et al., Phys. Rev. B 48, 15584 (1993).

[28] P.E. Blöchl and J.H. Stathis, Phys. Rev. Lett. 83, 372 (1999).

[29] P. E. Blöchl, Phys. Rev. B 62, 6158 (2000).

[30] T. Schroeder et al., Phys. Rev. B 66, 165422 (2002).

[31] U. Martinez, L. Giordano, and G. Pacchioni, Phys. Chem. B 110, 17015 (2006). 